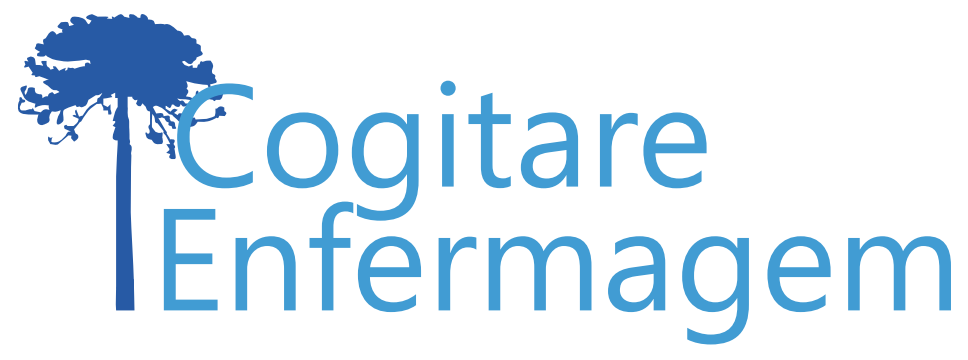

\title{
PRESCRIÇÃO E USO DE METRONIDAZOL PARA CONTROLE DO ODOR EM FERIDAS NEOPLÁSICAS
}

Nauã Rodrigues de Souza1, Maria Theresa Camilo de Lima², Rayanne Paullyne da Silva Batista ${ }^{3}$ Aline Milany da Silva Santos ${ }^{4}$, Magaly Bushatsky ${ }^{5}$, Isabel Cristina Ramos Vieira Santos ${ }^{6}$

\section{RESUMO}

Objetivo: verificar os aspectos relacionados à prescrição, preparo e administração do metronidazol para controle do odor em feridas neoplásicas.

Metodologia: estudo transversal, com 80 profissionais de saúde de cinco hospitais referência em oncologia em Recife-PE, entre agosto e outubro de 2017. Foram analisadas as variáveis: caracterização profissional, critérios para prescrição, apresentação, diluição, aplicação, frequência e cuidados na aplicação. Calculou-se média e desvio padrão para as variảveis racionais discretas, e para categóricas dicotômicas, teste qui-quadrado com correção de Yates.

Resultados: os enfermeiros caracterizaram-se pelo pouco tempo de experiência (1-3 anos), menor frequência de especialização comparados aos médicos, porem maior frequência de atualização em cuidados paliativos. Quanto à prescrição e utilização do produto, observou-se prescriçỗes alternativas e empíricas, com maceração de comprimidos 14 (53,8\%) ou solução injetável em cinco $(19,3 \%)$.

Conclusão: resultados evidenciam a escassa literatura sobre a temática e levantam a necessidade de construção de protocolos fundamentados em evidências científicas.

DESCRITORES: Enfermagem oncológica; Ferimentos e Lesões; Anti-Infecciosos; Metronidazol; Odorantes.

COMO REFERENCIAR ESTE ARTIGO:

Souza NR, Lima MTC, Batista RPS, Santos AMS, Bushatsky M, Santos ICRV. Prescrição e uso de metronidazol para controle do odor em feridas neoplásicas. Cogitare enferm. [Internet]. 2019 [acesso em "colocar data de acesso, dia, mês abreviado e ano"]; 24. Disponível em: http://dx.doi.org/10.5380/ce.v24i0.57906.

Este obra está licenciado com uma Licença Creative Commons Atribuição 4.0 Internacional.

${ }^{1}$ Enfermeiro. Mestre em Enfermagem. Universidade de Pernambuco. Recife, PE, Brasil. (?)

${ }^{2}$ Enfermeira. Universidade de Pernambuco. Recife, PE, Brasil.

${ }^{3}$ Discente de Enfermagem. Universidade de Pernambuco. Recife, PE, Brasil. 9

${ }^{4}$ Discente de Enfermagem. Universidade de Pernambuco. Recife, PE, Brasil. 9

${ }^{5}$ Enfermeira. Doutora em Enfermagem. Docente da Universidade de Pernambuco. Recife, PE, Brasil. ()

${ }^{6}$ Enfermeira. Doutora em Saúde Pública. Docente de Pós-Graduação em Enfermagem da Universidade de Pernambuco.

Recife, PE, Brasil. 9 


\title{
PRESCRIPTION AND USE OF METRONIDAZOLE FOR THE CONTROL OF ODOR IN NEOPLASTIC WOUNDS
}

\begin{abstract}
Objective: To check aspects related to the prescription, preparation and administration of metronidazole for the control of odor in neoplastic wounds.

Methodology: Cross-sectional study with 80 health professionals from five reference hospitals in oncology in Recife-PE, between August and October 2017. The following variables were analyzed: professional characterization, criteria for prescription, presentation, dilution, application, frequency and care in the application. Mean and standard deviation were calculated for obtaining the discrete rational variables, and chi-square test with Yates correction was used for dichotomous categorical variables.

Results: The nurses had little professional experience (1-3 years), less specialization compared to physicians, but had greater expertise in palliative care. Regarding the prescription and use of the product, 14 (53.8\%) professionals used alternative and empirical prescriptions, with maceration of tablets, and five (19.3\%) used injectable solution.

Conclusion: According to the results obtained, there are few studies on the subject and protocols based on scientific evidence should be constructed.
\end{abstract}

DESCRIPTORS: Oncologicalnursing; Wounds and Injuries; Anti-Infective agents; Metronidazole; Odorants.

\section{ASPECTOS ASOCIADOS A LA PRESCRIPCIÓN Y USO DE METRONIDAZOL EN EL CONTROL DEL OLOR EN HERIDAS NEOPLÁSICAS}

\section{RESUMEN:}

Objetivo: verificar los aspectos asociados a la prescripción, preparación y administración del metronidazol para control del olor en heridas neoplásicas.

Metodología: estudio transversal, con 80 profesionales de salud de cinco hospitales referencia en oncología en Recife, PE, entre agosto y octubre de 2017. Se analizaron las variables: caracterización profesional, criterios para prescripción, presentación, dilución, aplicación, frecuencia y cuidados en la aplicación. Se calcularon el promedio y la desviación típica para las variables racionales discretas, y para categóricas dicotómicas, test chi cuadrado con corrección de Yates.

Resultados: los enfermeros se caracterizaron por el poco tiempo de experiencia (1-3 años), menor frecuencia de especialización en comparación con los médicos, pero con más frecuencia de actualización en cuidados paliativos. Acerca de la prescripción y uso del producto, se observaron prescripciones alternativas y empíricas, con maceración de pastillas 14 (53,8\%) o solución inyectable en cinco (19,3\%).

Conclusión: resultados evidencian la escasa literatura sobre la temática y apuntan a la necesidad de construcción de protocolos basados en evidencias científicas.

DESCRIPTORES: Enfermería oncológica; Heridas y lesiones; Anti-Infecciosos; Metronidazol; Odorantes. 
As feridas neoplásicas são definidas como uma infiltração do tumor ou a metástase na pele que pode envolver vasos sanguíneos e linfáticos aferentes e são mais comuns em pacientes com câncer de mama, embora também apresente alta incidência em pacientes com câncer de cabeça e pescoço ${ }^{(1,2)}$.

A prevalência destas feridas é desconhecida devido à carência de estudos de base populacional. No entanto, estima-se que as feridas neoplásicas acometam $5 \%$ dos pacientes em estágio avançado da doença e 10\% dos pacientes com metástase, com uma expectativa de vida média de 6 a 12 meses $^{(2,3)}$.

Estas lesões neoplásicas apresentam uma grande quantidade de sintomas físicos, como odor fétido, dor, prurido, exsudato e sangramentos. Dentre estes, o odor, muitas vezes descrito por pacientes, membros da família e outros cuidadores incluindo enfermeiros, como semelhante a lixo, carne podre e cheiro de cadáver, é atribuído à proliferação de bactérias aeróbicas e anaeróbicas, as últimas das quais prosperam em tecido necrótico, livre de oxigênio, encontrado no leito destas feridas ${ }^{(4)}$ e contribuem substancialmente para a baixa autoestima, isolamento social e diminuição da qualidade de vida.

Intervenções voltadas a minorar o sofrimento causado pelo odor, dentre outros sintomas físicos e psicológicos, estão inseridas no âmbito dos cuidados paliativos e visam assegurar a melhor qualidade de vida possível, devendo estar baseadas nas melhores evidências, equilibrando os objetivos do cuidado aos recursos disponíveis ${ }^{(4)}$.

Ainda que o odor seja o sintoma que mais deprecia o paciente psicossocialmente, há insuficientes evidências a respeito da terapia tópica ideal para seu controle. Diversos produtos tópicos têm sido utilizados, destacando-se o metronidazol, classificado como antimicrobiano derivado do imidazólico que atua diretamente no ácido desoxirribonucleico (DNA) dos micro-organismos, impedindo a síntese de enzimas essenciais à sobrevida do patógeno e assim controlando indiretamente o odor ${ }^{(5)}$.

No Brasil tem sido recomendado o uso do metronidazol gel a 0,8\% nas feridas neoplásicas por apresentar excelentes resultados no controle do odor, sem a indução dos efeitos colaterais da terapia sistêmica e, na indisponibilidade deste, tem sido proposto alternativamente a utilização por via tópica de comprimidos macerados ou da solução injetável pura. ${ }^{(6)}$ Apesar disto, alguns estudos de revisão demonstram a falta de evidênçias robustas que recomendem o uso tópico, devido a questões como pequenos tamanhos de amostra e métodos de randomização, gerando incertezas sobre a validade dos resultados, ${ }^{(5-7)}$ assim como problemas relacionados a requisitos especiais de prescrição que podem influenciar seu uso(8).

Também não se verifica consenso sobre a forma de apresentação indicada ou o modo de uso com base na comparação de efetividade e custo e, desta maneira, cada instituição prepara formulações tópicas diferentes de metronidazol para pacientes com feridas neoplásicas ${ }^{(9)}$. Para elaboração de protocolos que sistematizem a assistência prestada ao paciente com ferida neoplásica, há necessidade de maior concentração de evidências científicas no Brasil.

Tendo em vista a necessidade de se conhecer como as instituições de saúde vêm utilizando o metronidazol para o controle do odor de feridas neoplásicas, para agregar evidencias e subsídios à padronização de condutas clínicas e construção de protocolos de cuidados, esta pesquisa objetivou verificar os aspectos relacionados à prescrição e uso do metronidazol para controle do odor em feridas neoplásicas.

\section{MÉTODO}

Trata-se de um estudo transversal, descritivo com abordagem quantitativa, 
desenvolvido em cinco hospitais de referência no tratamento de oncologia localizados na cidade de Recife. A população foi constituída por 80 participantes, sendo 51 enfermeiros e 29 médicos.

Os critérios de inclusão foram: profissionais de saúde, médicos e enfermeiros, das unidades clínicas de oncologia dos hospitais participantes. Foram excluídos aqueles que estivessem de férias ou em algum tipo de licença no período da coleta, além dos que trabalhassem no período noturno.

A coleta ocorreu no período de agosto a outubro de 2017, com dois questionários elaborados pelos pesquisadores, especificamente para cada categoria profissional, contendo as variáveis de interesse: caracterização do profissional; conhecimento sobre controle do odor; critérios de prescrição, forma de apresentação, forma de aplicação, frequência e cuidados observados na aplicação.

Os dados foram tabulados através do programa SPSS versão 21 e analisados através de estatística descritiva, com distribuição de frequência simples e absoluta. Para as variáveis racionais discretas, foram calculadas: média $(\bar{x})$, mediana $(\tilde{x})$ e desvio padrão $(a)$, enquanto para variáveis categóricas dicotômicas foi utilizado o teste qui-quadrado $(x 2)$ com correção de continuidade de Yates, assumindo-se um nível de significância de 5\% $(p<0,05)$.

Este estudo atendeu aos princípios éticos, a pesquisa foi aprovada pelo Comitê de Ética em Pesquisa do Complexo Hospitalar da Universidade de Pernambuco (HUOC) PROCAPE) com o Parecer $n^{\circ}$ 2.332.974.

\section{RESULTADOS}

A Tabela 1 apresenta a caracterização dos profissionais que participaram da pesquisa. Nota-se uma maior frequência de enfermeiros $23(45,1 \%)$ com faixa etária entre $31-40$ anos $(\bar{x}: 34,5 ; \alpha: 6,9 ; \tilde{x}: 34,0)$. Observou-se maior frequência de médicos na mesma faixa etária 18 $(62,1 \%)(\bar{x}: 34,9 ; a: 6,4 ; \tilde{x}: 34,0)$.

Tabela 1 - Caracterização da amostra. Recife, PE, Brasil, 2017 (continua)

\begin{tabular}{lcc} 
Variável & Enfermeiros (\%) & Médicos (\%) \\
\hline Faixa etária & & \\
\hline $20-30$ anos & $19(37,3)$ & $8(27,6)$ \\
\hline $31-40$ anos & $23(45,1)$ & $18(62,1)$ \\
\hline $41+$ anos & $9(17,6)$ & $3(10,3)$ \\
\hline Tempo de experiência & & \\
\hline $1-3$ anos & $30(58,8)$ & $16(55,2)$ \\
\hline $3-6$ anos & $18(35,3)$ & $5(17,2)$ \\
\hline $6 \geq$ anos & $3(5,9)$ & $8(27,6)$ \\
\hline Especialização em oncologia & & \\
\hline Sim & $22(43,1)$ & $23(79,3)$ \\
\hline Não & $29(56,9)$ & $6(20,7)$ \\
\hline Atualização em cuidados paliativos & & \\
\hline Sim & $27(52,9)$ & $14(48,3)$ \\
\hline Não & $24(47,1)$ & $15(51,7)$ \\
\hline
\end{tabular}




\begin{tabular}{lll}
\hline Controle do odor & & \\
\hline Sim & $28(54,9)$ & $10(34,5)$ \\
\hline Não & $23(45,1)$ & $19(65,5)$
\end{tabular}

Quanto ao tempo de experiência em cada profissão, 30 enfermeiros (58,8\%) $(\bar{x}: 3,3$; $\alpha: 2,3 ; \tilde{x}: 3,0)$ e $16(55,2 \%)$ médicos $(\bar{x}: 5,1 ; \alpha: 5,0 ; \tilde{x}: 2,0)$ tinham de um a três anos, sendo que a maior frequência destes últimos $23(79,3 \%)$ possuíam especialização em oncologia, ao contrário do verificado para os enfermeiros, e esta diferença foi significativa.

Quanto à abordagem do "Controle de Odor" durante as atualizações, 28 (54,9\%) enfermeiros referiram a presença do tema.

A Figura 1 mostra os produtos conhecidos por enfermeiros e médicos para o controle do odor das lesões neoplásicas. O metronidazol foi referido com maior frequência por ambos os grupos $41(80,4 \%)$ e $24(82,7 \%)$, respectivamente, seguido pelo carvão ativado $21(41,2 \%)$ e sete $(24,1 \%)$; e sulfadiazina de prata $18(35,3 \%)$ e três $(10,3 \%)$.

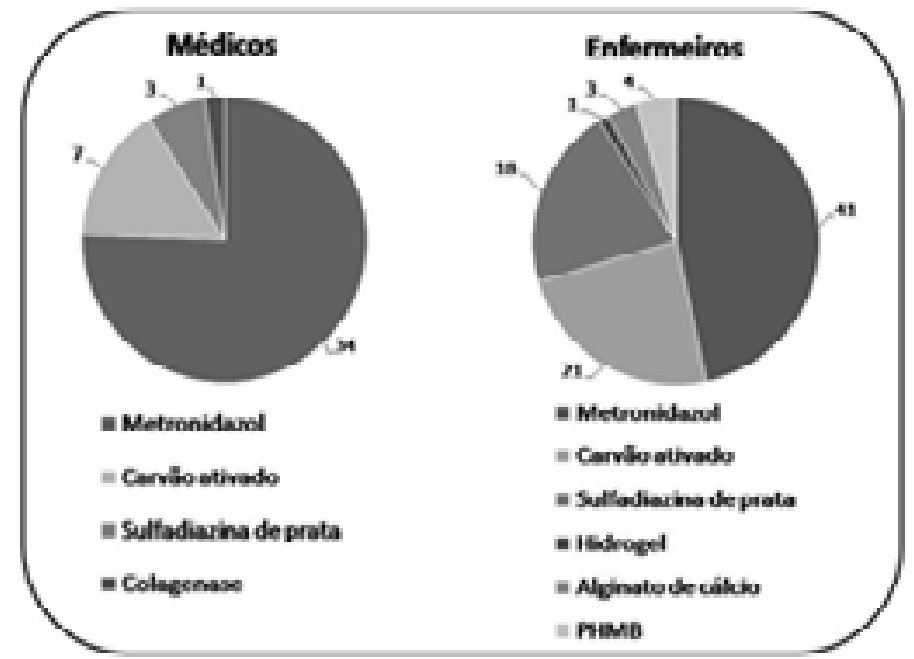

Figura 1 - Conhecimento dos produtos para controle do odor por enfermeiros e médicos. Recife, PE, Brasil, 2017

A Tabela 2 apresenta os critérios para a prescrição do metronidazol e utilização da droga. Foi observado que a maior frequência de médicos $12(15 \%)$ prescrevem o antimicrobiano de acordo com a disponibilidade do produto. A forma de apresentação mais prescrita foi a de comprimidos macerados $14(53,8 \%)$.

Tabela 2 - Critérios para prescrição e uso de metronidazol. Recife, PE, Brasil, 2017 (continua)

\begin{tabular}{lc} 
Variável & n (\%) \\
\hline Critérios para prescrição & \\
\hline Disponibilidade do produto & $12(15)$ \\
\hline Avaliação clínica & $11(13,8)$ \\
\hline Indicação da enfermagem & $2(2,5)$ \\
\hline
\end{tabular}




\begin{tabular}{lc}
\hline \multicolumn{2}{l}{ Forma de apresentação prescrita } \\
\hline Comprimidos macerados & $14(53,8)$ \\
\hline Gel & $7(26,9)$ \\
\hline Solução injetável & $5(19,3)$ \\
\hline Diluição da solução injetável \\
\hline $10 \mathrm{mg} / 100 \mathrm{ml}$ & $2(40)$ \\
\hline $500 \mathrm{mg} / 100 \mathrm{ml}$ & $2(40)$ \\
\hline $15 \mathrm{mg} / 100 \mathrm{ml}$ & $1(20)$
\end{tabular}

Na Tabela 3, encontram-se os resultados obtidos da análise do modo de uso do metronidazol por enfermeiros. Nesta, observa-se que a forma de aplicação da droga, referida por $20(43,5 \%)$ enfermeiros, se dá diretamente no leito da ferida, enquanto que a mesma frequência aplica o produto com o auxílio de gaze umedecida. A frequência de utilização da droga mais destacada foi de 2 vezes ao dia por $21(51,2 \%)$ enfermeiros, enquanto que $19(46,3 \%)$ deles referiu a aplicação 1 vez ao dia, conforme prescrição médica. Em relação aos cuidados observados na aplicação, destaca-se que 17 (34,7\%) realizam limpeza da ferida com soro fisiológico morno antes da aplicação do metronidazol.

Tabela 3 - Modo de uso do metronidazol por enfermeiros. Recife, PE, Brasil, 2017

\section{Variável}

$\mathbf{N}(\%)$

Forma de aplicação

Aplicação diretamente no leito da ferida $20(43,5)$

Em gaze umedecida $20(43,5)$

Irrigação da lesão

Frequência de aplicações

\begin{tabular}{lc}
\hline 1x dia & $19(46,3)$ \\
\hline 2x dia & $21(51,2)$ \\
\hline De 2 em 2 dias & $1(2,4)$ \\
\hline Cuidados observados na aplicação & $17(34,7)$ \\
\hline Realiza limpeza da ferida com SF morno antes da aplicação do metronidazol & $12(24,5)$ \\
\hline Após a aplicação do metronidazol, ocluir com gaze seca & $12(24,5)$ \\
\hline Utiliza o metronidazol juntamente com outros produtos/coberturas & $2(4,1)$
\end{tabular}

\section{DISCUSSÃO}

A oncologia é uma área complexa, que exige dos profissionais um olhar crítico e embasamento científico para compreender as circunstâncias e particularidades de cada paciente e sua situação clínica. O tempo de atuação dos profissionais influencia 
diretamente no conhecimento e experiência deles, porém, são fundamentais a capacitação e a atualização acerca das inovações tecnológicas e científicas que podem auxiliá-los na tomada de decisões e manejo de diversas situações, melhorando a qualidade da assistência prestada ${ }^{(10)}$.

No que diz respeito aos enfermeiros, o tempo de atuação foi inferior ao encontrado em um estudo sobre cuidados paliativos realizado no Sudeste ${ }^{(11)}$. Embora não exista uma definição sobre tempo de atuação ideal para se alcançar expertise em enfermagem, os anos de experiência constituem um fator importante para a qualidade dos cuidados prestados. No entanto, importa salientar que é uma condição necessária, mas não suficiente, uma vez que os anos de experiência podem proporcionar fluidez e flexibilidade, mas não o pensamento reflexivo que é necessário à expertise ${ }^{(12)}$.

Atrelado a isto, os médicos em maior frequência possuíam especialização em oncologia, diferente do verificado para enfermeiros, e esta diferença foi estatisticamente significativa. No entanto, os enfermeiros em maior frequência haviam cursado atualização em cuidados paliativos em que foi abordado o tema controle do odor.

O conhecimento em cuidados paliativos é imprescindível para os profissionais que tratam de pacientes com feridas neoplásicas, tendo em vista que estas tem limitada possibilidade de cura e a atenção de saúde deve então estar voltada para: o controle dos sintomas dentre os quais o odor, conforto, prevenção de agravos e incapacidades, promoção da independência e autonomia, ativação de recursos emocionais e sociais de enfrentamento do processo de adoecimento e terminalidade, e apoio e orientação à família e cuidadores ${ }^{(13)}$.

Estudos chamam atenção do conhecimento em oncologia limitado na graduação e das lacunas existentes, proporcionando aos egressos dificuldades na prestação de cuidados específicos do setor ${ }^{(14,15)}$. Por outro lado, o fato da maior frequência dos enfermeiros que participaram deste estudo haverem cursado atualização em cuidados paliativos exemplifica a necessidade percebida por eles de capacitar-se melhor para atender à demanda.

O crescimento atual e projetado para sobrevida de câncer exige maior atenção ao desenvolvimento de enfermeiros em oncologia. Quanto a isto, o conhecimento de enfermagem irá permitir-lhes justificar ações ou mesmo evitar o uso de práticas inseguras. Para isto, existe o desafio adicional da necessidade de desenvolvimento profissional contínuo, uma vez que o conhecimento de enfermagem está em constante evolução, e a expectativa é a de basear-se na melhor evidência para fornecer o cuidado mais apropriado para cada paciente ${ }^{(16)}$.

A necessidade de conhecimento científico na área de cuidados de pacientes com feridas é destacada por vários autores, no sentido da busca da qualidade da assistência, pois é uma área na qual a rápida evolução de produtos e coberturas mudou consideravelmente as práticas de assistência, mesmo considerando as feridas neoplásicas, e deu origem a novas recomendações profissionais ${ }^{(11,17)}$.

A literatura aponta que o conhecimento científico dos princípios dos cuidados paliativos é essencial para o processo de cuidar de pacientes oncológicos que apresentam feridas. De fato, como a perspectiva de cura dessas lesões é remota, o cuidado paliativo focaliza dois pontos cruciais de abordagem: físico e psicológico. No entanto, é apontado que as feridas neoplásicas geram os maiores níveis de sintomas físicos entre todas as classes de feridas e, desta forma, o cuidado dos sintomas físicos é importante porque pode repercutir sobre as manifestações psicológicas do paciente, melhorando assim a autoestima e restaurando sua dignidade ${ }^{(18)}$.

Diversas técnicas e produtos foram desenvolvidos para auxiliar no tratamento das feridas neoplásicas. Entretanto, a terapia tópica específica ainda é questionável. Dentre os diversos produtos utilizados, o metronidazol é um dos principais, por ser um antibiótico com propriedades anti-inflamatórias e suprimir as células mediadoras do sistema imunológico. A literatura registra seu efeito de diminuição do odor a partir das primeiras vinte e quatro 
horas da utilização do produto, mantendo-se, pelo menos, até duas semanas seguintes ${ }^{(19)}$. Isto pode ter justificado a maior frequência de resposta por parte de médicos e enfermeiros quando questionados sobre produtos conhecidos para controle do odor.

Estudos indicam a aplicação de metronidazol gel a 0,75 ou $0,8 \%$ no leito da ferida, no entanto, como no Brasil não existem formulações prontas desta droga tópica a $0,8 \%$, torna-se necessária sua manipulação. Este fato pode ter influenciado os critérios adotados pelos médicos para prescrição, que neste estudo, obedeciam primeiro à disponibilidade do produto seguido pela avaliação clínica. Da mesma forma, pode também estar relacionado à forma prescrita, em maior frequência através de comprimidos macerados. Esta forma de uso atende ao preceituado pelo Ministério da Saúde do Brasil(5,8,9).

No que diz respeito à forma prescrita da solução injetável, não se observou homogeneidade nas respostas, que variaram de diluições de $10 \mathrm{mg} / 100 \mathrm{ml}$ até 500 $\mathrm{mg} / 100 \mathrm{ml}$ de SF. Esta prática vai de encontro às recomendações clínicas para o uso de solução injetável que deve ser administrada pura, sem diluir ${ }^{(6)}$.

Quanto ao modo de uso, os enfermeiros pesquisados referiram, em maior frequência, aplicar o metronidazol diretamente no leito da ferida ou por meio de gaze umedecida, duas vezes ao dia, após limpeza com solução fisiológica 0,9\% morna. Segundo um estudo, sobre a prática atual da gestão do odor da ferida realizada com 1.444 profissionais de 36 países, falta um protocolo padronizado para o modo, dose e frequência de aplicação do metronidazol. Desta forma, cada instituição prepara formulações tópicas diferentes, tornando a quantidade de droga usada nessas práticas impossível de determinar. As preparações comerciais variam de $0,75 \%$ a $0,8 \%$, no entanto, clinicamente, não está claro qual a quantidade de preparação deve ser usada em uma ferida para atingir uma concentração mínima do medicamento para um efeito terapêutico ${ }^{(20)}$.

Apesar das limitações relacionadas à metodologia de corte transversal, os resultados deste estudo são importantes, na medida em que apontam para questões que devem ser refletidas por gestores hospitalares e instituições formadoras, assim como pelos profissionais preocupados coma melhoria da assistência à população acometida por essa patologia.

\section{CONCLUSÃO}

Os profissionais responsáveis pela prescrição e uso do metronidazol para o controle do odor em feridas neoplásicas caracterizaram-se pelo pouco tempo de experiência e baixa qualificação a título de especialização em oncologia, no que diz respeito aos enfermeiros, embora os mesmos se apresentem mais capacitados quanto aos aspectos dos cuidados paliativos.

No que diz respeito à pratica de prescrição e aplicação do produto, os resultados demonstraram que está associada primeiramente à disponibilidade do produto, sendo prescrito de forma alternativa e empírica através da maceração de comprimidos ou diluição de solução injetável.

Estes resultados evidenciam que a escassa literatura sobre a temática levanta a necessidade de construção de protocolos fundamentados em evidências científicas, de modo a assegurar aos pacientes uma aplicação segura e efetiva.

\section{REFERÊNCIAS}

1. Gibson S, Green J. Review of patients experiences with fungating wounds and associated quality of life. J Wound Care [Internet]. 2013 [acesso em 07 out 2017]; 22(5) Disponível em: https://doi. org/10.12968/jowc.2013.22.5.265. 
2. Probst S, Arber A, Faithfull S. Coping with an exulcerated breast carcinoma: an interpretative phenomenological stud. J Wound Care [Internet]. 2013 [acesso em 07 out 2017]; 22(7). Disponível em: https://doi.org/10.12968/jowc.2013.22.7.352.

3. Agra G, Fernandes MA, Platel ICS, Freire MEM. Cuidados Paliativos ao Paciente Portador de Ferida Neoplásica: uma Revisão Integrativa da Literatura. Rev. bras. cancerol [Internet]. 2013 [acesso em 09 out 2017]; 59(1). Disponível em: http://www1.inca.gov.br/rbc/n_59/v01/pdf/16-cuidados-paliativos-aopaciente-portador-de-ferida-neoplasica.pdf.

4. Tilley C, Lipson J, Ramos M. Palliative wound care for malignant fungating wounds: holistic considerations at end-of-life. Nurs. Clin. North Am. [Internet]. 2016 [acesso em 20 out 2017]; 51(3). Disponível: https://doi. org/10.1016/j.cnur.2016.05.006.

5. de Castro DLV, Santos VLCG. Controlling wound odor with metronidazole: a systematic review. Rev. Esc. Enferm. USP. [Internet]. 2015 [acesso em 20 out 2017]; 49(5) Disponível em: http://dx.doi.org/10.1590/ S0080-623420150000500021.

6. Instituto Nacional de Câncer (INCA). Tratamento e controle de feridas tumorais e úlceras por pressão no câncer avançado: série cuidados paliativos. Rio de Janeiro: Instituto Nacional de Câncer; 2009.

7. Adderley UJ, Holt IGS. Topical agents and dressings for fungating wounds. Cochrane Database Syst Rev [Internet]. 2014 [acesso em 25 out 2017]; 15(5). Disponível em: http://dx.doi.org/10.1002/14651858. CD003948.pub3.

8. Akhmetova A, Saliev T, Allan IU, Illsley MJ, Nurgozhin T, Mikhalovsky S. A Comprehensive Review of Topical Odor-Controlling Treatment Options for Chronic Wounds. J Wound Ostomy Continence Nurs [Internet]. 2016 [acesso em 05 nov 2017]; 43(6). Disponível em: http://dx.doi.org/ 10.1097/WON.0000000000000273.

9. Watanabel K, Shimo A, Tsugawa K, Yutaka T, Yamauchi H, Miyai E, et al. Safe and effective deodorization of malodorous fungating tumors using topical metronidazole $0.75 \%$ gel (GK567): a multicenter, open-label, phase III study (RDT.07.SRE.27013). Support Care Cancer. [Internet]. 2016 [acesso em 05 nov 2017]; 24(6). Disponível em: http://dx.doi.org/10.1007/s00520-015-3067-0.

10. Souza AML, Freire DA, Santos ICRV, Melo JTS, Souza MAO, Souza NR. Perfil dos enfermeiros no serviço de oncologia e a importância da qualificação profissional. Nursing [Internet]. 2017 [acesso em 05 nov 2017]; 20(233). Disponível em: http://bases.bireme.br/cgi-bin/wxislind.exe/iah/online/?/sisScript=iah/iah. xis\&src=google\&base=BDENF\&lang=p\&nextAction=lnk\&exprSearch=31610\&indexSearch=ID.

11. Silva MM, Santana NGM, Santos MC, Cirilo JD, Barrocas DLR, Moreira MC. Cuidados paliativos na assistência de alta complexidade em oncologia: percepção de enfermeiros. Esc. Anna Nery [Internet]. 2015 [acesso em 05 nov 2017]; 19(3). Disponível em: http://dx.doi.org/10.5935/1414-8145.20150061.

12. Amaral AFS, Ferreira PL. Adaptação e validação da Clinical Nursing Expertise Survey para a população de enfermeiros portugueses. Esc. Anna Nery [Internet]. 2014 [acesso em 05 nov 2017]; 18(3). Disponível em: http://dx.doi.org/10.5935/1414-8145.20140070.

13. Gomes ALZ, Othero MB. Cuidados paliativos. Estud. av. [Internet]. 2016 [acesso em 05 nov 2017]; 30(88). Disponível em: http://dx.doi.org/10.1590/S0103-40142016.30880011.

14. Lockhart JS, Galioto M, Oberleitner MG, Fulton JS, McMahon D, George K, et al. A national survey of oncology content in prelicensure registered nurse programs. J Nurs Educ [Internet]. 2013 [acesso em 05 nov 2017]; 52(7). Disponível em: https://doi.org/10.3928/01484834-20130529-01.

15. Santos FC, Camelo SHH, Laus AM, Leal LA. O enfermeiro que atua em unidades hospitalares oncológicas: perfil e capacitação profissional. Enferm. glob [Internet]. 2015 [acesso em 05 nov 2017]; 14(2). Disponível em: https://doi.org/10.6018/eglobal.14.2.190061.

16. Ginex PK, Hernandez M, Vrabel M. A Clinical Librarian-Nursing Partnership to Bridge Clinical Practice and Research in an Oncology Setting. Oncol Nurs Forum [Internet]. 2016 [acesso em 05 nov 2017]; 43(5). Disponível em: http://dx.doi.org/10.1188/16.ONF.549-552. 
17. Castro MC, Cruz P, Grellmann M, Santos W, Fuly P. Cuidados paliativos a pacientes com feridas oncológicas em Hospital universitário: relato de experiência. Cogitare enferm. [Internet]. 2014 [acesso em 07 nov 2017]; 19(4). Disponível em: http://dx.doi.org/10.5380/ce.v19i4.37294.

18. Kelechi TJ, Prentice M, Madisetti M, Brunette G, Mueller M. Palliative care in the management of pain, odor and exudate in chronic wounds at the end of life: a cohort study. J Hosp Palliat Nurs [Internet] 2017 [acesso em 29 out 2018]; 19(1). Disponível em: http://dx.doi.org/10.1097/NJH.0000000000000318.

19. Azevedo IC, Costa RKS, Torres GV, Ferreira Júnior MA. Tratamento de Feridas: A especificidade das lesões oncológicas. Revista Saúde e Pesquisa [Internet] 2014;7(2) [acesso em 10 nov 2017]. Disponível: http://periodicos.unicesumar.edu.br/index.php/saudpesq/article/view/3306/2380.

20. Gethin G, Grocott P, Probst S, Clarke E. Current practice in the management of wound odour: An international survey. Int J Nurs Stud [Internet]. 2014 [acesso em 10 nov 2017]; 51(6). Disponível em: http:// dx.doi.org/10.1016/j.ijnurstu.2013.10.013.

Recebido: 16/02/2018

Finalizado: 15/01/2019

Autor Correspondente:

Isabel Cristina Ramos Vieira Santos

Universidade de Pernambuco

R. Arnóbio Marquês, 310 - 50100-130 - Recife, PE, Brasil

E-mail: tutornad@yahoo.com.br

Contribuição dos autores:

Contribuições substanciais para a concepção ou desenho do estudo; ou a aquisição, análise ou interpretação de dados do estudo - NRS, MTCL, RPSB, AMSS, MB, ICRVS

Elaboração e revisão crítica do conteúdo intelectual do estudo - MB

Responsável por todos os aspectos do estudo, assegurando as questões de precisão ou integridade de qualquer parte do estudo - ICRVS 